\title{
Nonlinear Vibration Analysis of a Rigid Rotating Shaft Supported by the Magnetic Bearing (Influence of the Integral Feedback in the PID Control of the Vertical Shaft)*
}

\author{
Tsuyoshi INOUE** and Yasuhiko SUGAWARA*** \\ ** Department of Mechanical Science and Engineering, School of Engineering, Nagoya University \\ Furo-cho,Chikusa-ku,Nagoya,Aichi 464-8603, Japan \\ E-mail: inoue@ nuem.nagoya-u.ac.jp \\ *** DAIHATSU MOTOR CO.,LTD. \\ 1-1 Daihatsu-cho, Ikeda, Osaka, 563-8651, JAPAN
}

\begin{abstract}
Active magnetic bearing (AMB) becomes to be widely used in various kinds of rotating machinery. However, as the magnetic force is nonlinear, nonlinear phenomena may occur when the rotational speed becomes higher and the delay of control force relatively increases. In this paper, the magnetic force is modeled by considering both the second order delay of the electric current and the first order delay of the magnetic flux. The AMB force is represented by a power series function of the electric current and shaft displacement. The nonlinear theoretical analysis of the vertical rigid rotor supported by the AMB with the PID control theory is demonstrated. The effects of the gain of the integral feedback on the nonlinear phenomena are clarified theoretically and experimentally.
\end{abstract}

Key words : Vibration of Rotating Body, Magnetic Bearing, Forced Vibration, Nonlinear Vibration

\section{Introduction}

In the active magnetic bearing $(\mathrm{AMB})^{(1)(2)}$, the magnetic force is essentially nonlinear. Chang et al. ${ }^{(3)}$ studied the modeling of the AMB considering the system nonlinearity, and identified the soft-spring type strong nonlinear characteristics. Therefore, the vibration phenomena, such as the sudden amplitude variation due to the jump phenomena or the occurrence of the sub-resonances, may occur in the rotating shaft supported by the AMB, and these are not able to be solved by the general linear analysis ${ }^{(4)-(9)}$. Thus, the clarification of the nonlinear vibration phenomena in the rotating shaft supported by the AMB is necessary for the prediction, diagnosis and prevention of the vibration problem in the practical system.

In the conventional studies on the vibration phenomena caused in the AMB, Chinta ${ }^{(4)}$ studied the nonlinear vibration of the rigid rotor supported by the AMB with PD control using numerical simulation. He explained the occurrence of the nonlinear vibration using the Floquet theory. Ji et al. ${ }^{(5)-(8)}$ expressed the characteristic of the AMB approximately in the cubic nonlinear function of both the displacement and the velocity, and investigated the various nonlinear vibration phenomena and bifurcation phenomena by the numerical simulation and the theoretical analysis. Ho et al.$^{(9)}$ performed the numerical simulation for the finite element model of the elastic rotating shaft supported by the AMB. He used the unbalance as the parameter, and investigated the bifurcation phenomena due to the characteristic of the thrust bearing by utilizing the Floquet theory. However, these investigated nonlinear phenomena in the AMB were not confirmed experimentally. Also, the validity of both its approximation of the magnetic force and the values of used parameters were not explained clearly. 


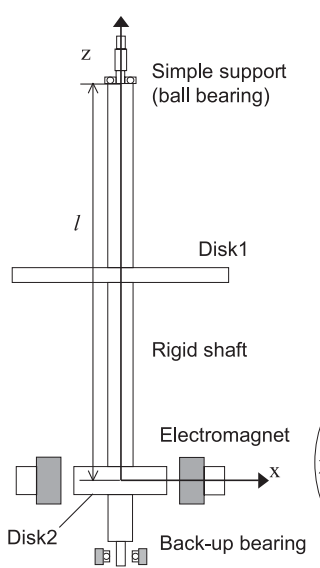

(a)

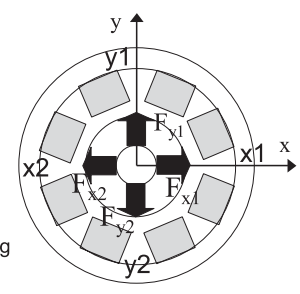

(b)

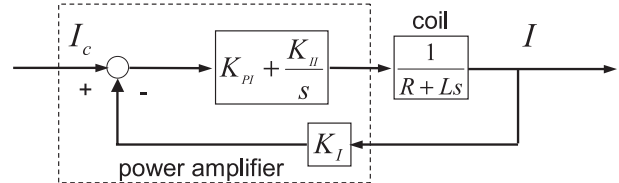

(c)

Fig. 1 Model of the rotor supported by the AMB and the block diagram of the amplifier

In the control of the AMB, the delay occurs due to the dynamics in the calculation unit, electric circuit part, and the magnetic circuit part, respectively ${ }^{(1)(2)}$. However, the influence of the interaction between these dynamics and the system nonlinearity on the vibration characteristic of the rotor system supported by the AMB has not been investigated.

In this paper, the rotating rigid shaft supported vertically by the AMB is considered. The current control type power amplifier is used, and its PI control characteristic and the delay of the magnetic flux in the electromagnet are considered. The modeling of the rotor system supported by the PID controlled AMB is performed.

There are many types of power operational amplifiers those supply only the positive electric current. Furthermore, in order to avoid the destabilization, the electromagnet current is usually not supplied when the reference (ordered) value becomes negative. In this paper, the case with no negative current supplied is considered, and it causes the discontinuous magnetic flux characteristic. Its discontinuous characteristic is approximated by the power series function, and the nonlinear theoretical analysis is performed. By the nonlinear theoretical analysis, the influence of the integral feedback control on the vibration characteristic is investigated. Its influence of the integral feedback control is also investigated experimentally, and the nonlinear vibration characteristic and the bifurcation phenomena are confirmed.

\section{Theoretical Model and Equations of Motion}

\subsection{Theoretical Model}

Figure 1(a) shows the theoretical model. The vertical rigid rotor is supported by the AMB at the lower end, and its upper end is simply supported by a ball bearing. Two disks, disk 1 and disk 2, are mounted on the shaft. The AMB consists of 4 elements, the displacement sensor, the controller, the power amplifier, and the electromagnets. The four electromagnets in the positive and negative sides of both the $x$ and $y$ axes are numbered as $x_{1}, x_{2}, y_{1}$, and $y_{2}$, respectively. The attraction force of the electromagnet is represented as ${ }^{(1)(2)}$ :

$$
f_{m}=\frac{B^{2}}{2 \mu_{0}} 2 S=k_{m} \frac{I^{2}}{(R+\delta)^{2}}[\mathrm{~N}]
$$

The notations $k_{m}=\mu_{0} S N^{2} / 4$ and $\delta=l_{m} / 2 \mu_{s}$ are the characteristic constants of magnetic force. Here, $B$ is the magnetic flux density, $N$ is the turn number of the coil, $I$ is the electric current, $\mu_{0}$ is the magnetic permeability in the vacuum, $\mu_{s}$ is the relative permeability of the electromagnet iron core, $l_{m}$ is the magnetic path length of the electromagnet, $S$ is the area of the electromagnet pole, and $R$ is the gap between the rotor and the electromagnet. 


\subsection{Equations of Motion for the Rotor System}

Electromagnetic forces of the electromagnets of $x_{1}, x_{2}, y_{1}$, and $y_{2}$ are expressed as $F_{x 1}$, $F_{x 2}, F_{y 1}$, and $F_{y 2}$, respectively. Forces $F_{x 1}$ and $F_{x 2}$ show the magnetic forces in the $x$ direction, and forces $F_{y 1}$ and $F_{y 2}$ show the magnetic forces in the $y$ direction.

In the system shown in Fig.1(a), the displacement angles, $\theta_{x}$ and $\theta_{y}$, of the shaft in the $x z$ and the $y z$ planes are introduced. Then, the equations of motion of the rotating shaft considering the effect of the gyroscopic moment are represented as:

$$
I_{d} \ddot{\theta}_{x}+I_{p} \omega \dot{\theta}_{y}=l\left(F_{x 1}-F_{x 2}\right)+\operatorname{lme} \omega^{2} \cos \omega t, I_{d} \ddot{\theta}_{y}-I_{p} \omega \dot{\theta}_{x}=l\left(F_{y 1}-F_{y 2}\right)+\operatorname{lme} \omega^{2} \sin \omega t
$$

where, $I_{d}$ is the diametral moment of inertia of the rotating shaft about the upper support point, $I_{p}$ is the polar moment of inertia, $m$ is the equivalent mass of the rotating shaft at disk 2 position, $l$ is the shaft length, $e$ is the equivalent static eccentricity at disk $2, \omega$ is the angular velocity, and $t$ is time. The second term in the left side represents the gyroscopic effect.

The displacement of disk 2 is represented by coordinate $(x, y)$. By using the notation of shaft length $l$ and the relationships $x=l \theta_{x}$ and $y=l \theta_{y}$, the equations of motion are arranged in terms of the shaft displacement $x$ and $y$ as:

$$
I_{d} \ddot{x}+I_{p} \omega \dot{y}=l^{2}\left(F_{x 1}-F_{x 2}\right)+l^{2} m e \omega^{2} \cos \omega t, I_{d} \ddot{y}-I_{p} \omega \dot{x}=l^{2}\left(F_{y 1}-F_{y 2}\right)+l^{2} m e \omega^{2} \sin \omega t
$$

\subsection{Governing equation of the magnetic flux}

It is assumed that magnetic flux saturation does not occur in the electromagnet core. When the rotating shaft is the equilibrium condition with both the gap $R_{0}$ and the bias electric current $I_{0}$, the bias magnetic flux density $B_{0}$ is derived from Eq.(1) as:

$$
B_{0}=\frac{\mu_{0} N I_{0}}{2\left(R_{0}+\delta\right)}
$$

The values of the electric current in the electromagnets are denoted as $I_{x 1}=I_{0}-i_{x}, I_{x 2}=I_{0}+i_{x}$, $I_{y 1}=I_{0}-i_{y}$, and $I_{y 2}=I_{0}+i_{y}$, respectively. Also, the variations of magnetic flux density from the bias value $B_{0}$ in electromagnets are denoted as $B_{x 1}, B_{x 2}, B_{y 1}$ and $B_{y 2}$, respectively.

It is known that the magnetic flux of electromagnet shows the characteristic which is similar to the first-order dynamical characteristic ${ }^{(1)(2)}$. In this paper, it is assumed that the variations of magnetic flux densities $B_{x 1}, \ldots, B_{y 2}$ from the bias value $B_{0}$ have the dynamical characteristics of the first-order. This delay of the magnetic flux is caused due to such as the eddy current in the electromagnet's core, and the coefficient of the delay is denoted as $\beta$. The governing equations of the magnetic flux of electromagnets are represented as follows ${ }^{(2)}$ :

$$
\begin{aligned}
& \beta \dot{B_{x 1}}+\left(B_{0}+B_{x 1}\right)=\frac{\mu_{0} N\left(I_{0}-i_{x}\right)}{2\left(R_{0}+\delta-x\right)}, \quad \beta \dot{B_{x 2}}+\left(B_{0}+B_{x 2}\right)=\frac{\mu_{0} N\left(I_{0}+i_{x}\right)}{2\left(R_{0}+\delta+x\right)} \\
& \left.\beta \dot{B_{y 1}}+\left(B_{0}+B_{y 1}\right)=\frac{\mu_{0} N\left(I_{0}-i_{y}\right)}{2\left(R_{0}+\delta-y\right)}, \quad \beta \dot{B_{y 2}}+\left(B_{0}+B_{y 2}\right)=\frac{\mu_{0} N\left(I_{0}+i_{y}\right)}{2\left(R_{0}+\delta+y\right)}\right\}
\end{aligned}
$$

\subsection{Governing equation of the electric current}

Next, the dynamical equations of the electric current variations, $i_{x}$ and $i_{y}$, are represented. In this modeling, the characteristics of a current-control-type power amplifier, which is used in the experiment, is taken into consideration. The slight delay is still caused between the input signal and the output electric current due to the amplifier's saturation and so on ${ }^{(2)}$. Figure 1(b) is the block diagram of the current-control-type power amplifier (HPtec, $\mu$ SC-4AT) used in the experiment. Symbols $K_{P I}, K_{I I}$ and $K_{I}$ are the gains of PI control and the gain of the electric current feedback in the power amplifier, respectively. Symbols $R$ and $L$ are the resistance and 
Table 1 Parameter values

\begin{tabular}{|c|c|c|c|c|c|}
\hline$K_{P I}$ & $15.0 \mathrm{~V} / \mathrm{V}$ & $K_{I I}$ & $606 \mathrm{~s}^{-1}$ & $K_{I}$ & $1.00 \mathrm{~V} / \mathrm{A}$ \\
\hline$k_{m}$ & $1.56 \times 10^{4} \mathrm{~kg} \mathrm{~mm}^{3} / \mathrm{A}^{2} \mathrm{~s}^{2}$ & $R_{0}$ & $0.80 \mathrm{~mm}$ & $\delta$ & $0.141 \mathrm{~mm}$ \\
\hline$I_{0}$ & $0.50 \mathrm{~A}$ & $I_{d}$ & $5.43 \times 10^{5} \mathrm{kgmm}$ & $l$ & $5.0 \times 10^{2} \mathrm{~mm}$ \\
\hline$I_{p}$ & $1.33 \times 10^{4} \mathrm{kgmm}^{2}$ & $m$ & $2.04 \mathrm{~kg}$ & $e$ & $2.93 \times 10^{-2} \mathrm{~mm}$ \\
\hline$\alpha_{1}$ & $3.79 \times 10^{-2} \mathrm{~s}$ & $\alpha_{2}$ & $2.29 \times 10^{-5} \mathrm{~s}^{2}$ & $\beta$ & $3.66 \times 10^{-3} \mathrm{~s}$ \\
\hline$N$ & 300 Turns & $p$ & $68.451 \mathrm{rad} / \mathrm{sec}$ & & \\
\hline
\end{tabular}

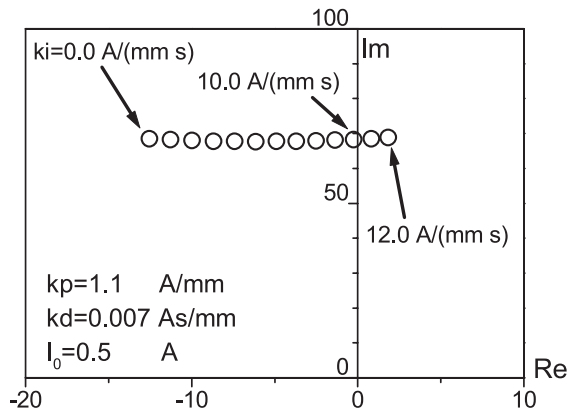

Fig. 2 Root locus for the integral gain $k_{i}$

the inductance of the electromagnet coil. The input signal $I_{c}$ and the output current $I$ of the power amplifier for the electromagnet $x 1$ are $I_{c}=K_{I}\left(I_{0}+k_{p} x+k_{d} \dot{x}+k_{i} \int x d t\right)$ and $I=I_{0}+i_{x}$, respectively. The parameters $k_{p}, k_{i}$ and $k_{d}$ are the feedback gains for proportional, integral, and derivative of the shaft displacement. Furthermore the variables $s_{x}$ and $s_{y}$, which hold the relationships $x=d s_{x} / d t$ and $y=d s_{y} / d t$, are introduced in order to represent the govening equations of the electric circuit with the PID control. From the block diagram, the governing equations of the electric current variations, $i_{x}$ and $i_{y}$, are derived as:

$$
\alpha_{2} \ddot{i_{x}}+\alpha_{1} \dot{i_{x}}+i_{x}=k_{i} s_{x}+k_{1} x+k_{2} \dot{x}+k_{3} \ddot{x}, \alpha_{2} \ddot{i_{y}}+\alpha_{1} \dot{i_{y}}+i_{y}=k_{i} s_{y}+k_{1} y+k_{2} \dot{y}+k_{3} \ddot{y}
$$

Here, the coefficients are represented as:

$$
k_{1}=k_{p}+\frac{K_{P I}}{K_{I I}} k_{i}, k_{2}=k_{d}+\frac{K_{P I}}{K_{I I}} k_{p}, k_{3}=\frac{K_{P I}}{K_{I I}} k_{d}, \alpha_{1}=\frac{R+K_{I} K_{P I}}{K_{I} K_{I I}}, \alpha_{2}=\frac{L}{K_{I} K_{I I}}
$$

\subsection{Dimensionless form}

Table 1 shows the dimensional parameter values of the experimental system. The set of linearized equations of Eqs.(3), (5) and (6) are used, and its eigenvalues for the parameter values shown in Table 1 are derived. The eigenvalue which is the nearest from the imaginary axis is focused, and Fig.2 shows its root locus for the integral feedback gain $k_{i}$. Based on this linear analysis, this rotating shaft system becomes unstable when the gain $k_{i}$ increases to about $10 \mathrm{~A} /(\mathrm{mms})$.

By using the coefficient of the electromagnet $k_{m}=\mu_{0} S N^{2} / 4$, by eliminating the time derivative terms in Eqs.(5) and (6), and by performing the system linearization of Eqs.(3) and (5) for the equilibrium position, the stiffness coefficient $k$ of the linearized system, $I_{d} \ddot{x}+$ $I_{p} \omega \dot{y}+k x=l^{2} m e \omega^{2} \cos \omega t$, is obtained as $k=4 l^{2} k_{m} I_{0}\left(k_{p}-I_{0} /\left(R_{0}+\delta\right)\right) /\left(R_{0}+\delta\right)^{2}$. The damped natural angular frequency $p_{d}$ for non-rotating condition with the integral feedback gain $k_{i}=0$ is derived from Fig.2. Then, the dimensionless forms of the system equations are derived by using the value of $p_{d}$, bias magnetic flux density $B_{0}$ obtained in Eq.(4), equilibrium gap $R_{0}$, 
and the bias electric current $I_{0}$. The dimensionless values are shown as:

$$
\begin{aligned}
& \dot{i_{p}}=I_{p} / I_{d}, \dot{\omega}=\omega / p_{d}, \dot{t}=t p_{d}, \dot{e}=l^{2} m e / I_{d} R_{0}, \dot{x}=x / R_{0}, \dot{y}=y / R_{0}, \dot{\delta}=\delta / R_{0}, \\
& \dot{s_{x}}=s_{x} p_{d} / R_{0}, \dot{s_{y}}=s_{y} p_{d} / R_{0}, \dot{i_{x}}=i_{x} / I_{0}, \dot{i}_{y}^{\prime}=i_{y} / I_{0}, \alpha_{1}^{\prime}=\alpha_{1} p_{d}, \alpha_{2}=\alpha_{2} p_{d}^{2}, \\
& \dot{\beta}=\beta p_{d} \dot{B}_{x 1}^{\prime}=B_{x 1} / B_{0}, B_{x 2}^{\prime}=B_{x 2} / B_{0}, B_{y 1}^{\prime}=B_{y 1} / B_{0}, B_{y 2}^{\prime}=B_{y 2} / B_{0}, \dot{k_{1}}=k_{1} R_{0} / p I_{0}, \\
& \dot{k_{2}}=k_{2} R_{0} / I_{0}, \dot{k_{3}}=k_{3} R_{0} p_{d} / I_{0}, \dot{k_{4}}=k_{4} R_{0} p_{d}^{2} / I_{0}, \hat{k}_{m}^{\prime}=l^{2} I_{0}^{2} k_{m} /\left\{k R_{0}\left(R_{0}+\delta\right)^{2}\right\}
\end{aligned}
$$

The dimensionless form of the governing equations, Eqs.(3), (5) and (6), are derived as follows:

$$
\left.\begin{array}{l}
\ddot{x}+i_{p} \omega \dot{y}=k_{m}\left\{\left(1+B_{x 1}\right)^{2}-\left(1+B_{x 2}\right)^{2}\right\}+e \omega^{2} \cos \omega t \\
\ddot{y}-i_{p} \omega \dot{x}=k_{m}\left\{\left(1+B_{y 1}\right)^{2}-\left(1+B_{y 2}\right)^{2}\right\}+e \omega^{2} \sin \omega t
\end{array}\right\}
$$

$$
\begin{aligned}
& \beta \dot{B}_{x 1}+\left(1+B_{x 1}\right) \\
& =\left\{\begin{array}{ll}
(1+\delta)\left(\frac{1-i_{x}}{1+\delta-x}\right) & \left(i_{x}<1\right) \\
0 & \left(i_{x}>1\right)
\end{array},= \begin{cases}(1+\delta)\left(\frac{1+i_{x}}{1+\delta+x}\right) & \left(i_{x}>-1\right) \\
0 & \left(i_{x}<-1\right)\end{cases} \right. \\
& \beta \dot{B}_{y 1}+\left(1+B_{y 1}\right) \\
& =\left\{\begin{array}{ll}
(1+\delta)\left(\frac{1-i_{y}}{1+\delta-y}\right) & \left(i_{y}<1\right) \\
0 & \left(i_{y}>1\right)
\end{array},= \begin{cases}(1+\delta)\left(\frac{1+i_{y 2}}{1+\delta+y}\right) & \left(i_{y}>-1\right) \\
0 & \left(i_{y}<-1\right)\end{cases} \right.
\end{aligned}
$$

$$
\alpha_{2} \ddot{i}_{x}+\alpha_{1} \dot{i}_{x}+i_{x}=k_{1} s_{x}+k_{2} x+k_{3} \dot{x}+k_{4} \ddot{x} \alpha_{2} \ddot{i}_{y}+\alpha_{1} \dot{i}_{y}+i_{y}=k_{1} s_{y}+k_{2} y+k_{3} \dot{y}+k_{4} \ddot{y}
$$

This paper considers the case with only the positive electric current in order to avoid the system destabilization. This discontinuity is represented in the equation of the magnetic flux density, Eq.(10). In the following analysis, these dimensionless equations, Eqs.(9)-(11), are used, and the symbol (') denoting the dimensionless value is omitted.

\subsection{Approximated Model of Magnetic Flux for the AMB}

Figures 3(a) and 3(b) show the magnetic flux densities, $\left(1+B_{x 1}\right)$ and $\left(1+B_{x 2}\right)$ in the static case $\left(\dot{B}_{x 1}=\dot{B}_{x 2}=0\right)$ of Eq.(10), and they show discontinuous characteristic. It is difficult to apply the conventional nonlinear analysis techniques to such a discontinuous nonlinear system. Thus, this paper develops the approximated representation using the continuous function.

The first representation is the direct one. Equation (10) is focused and approximated in the power series function of both the electric current $i_{x}$ and the shaft displacement $x$ as follows.

$$
1+B_{x 1}+\beta \dot{B_{x 1}}=1+P_{10} x+P_{01} i_{x}+P_{20} x^{2}+P_{11} x i_{x}+P_{02} i_{x}^{2}+P_{30} x^{3}+P_{21} x^{2} i_{x}+\cdots
$$

The coefficients $P_{i j}$ are determined by using the least square method. Figure 3(c) shows the approximate planes of $\left(1+B_{x 1}\right)$ by using the 5 th power series function. The usage of the higher order power series function gives the more accurate approximation, while it results in the more complex flow of the theoretical analysis. The accuracy of approximation is investigated, and it is clarified that at least the 7 th order power series function is required for the accurate approximation. In the case of the 7th order power series function, 35 terms appear for each electromagnet, and the total number of the terms for the AMB attains 140 which is large number for the nonlinear theoretical analysis. 


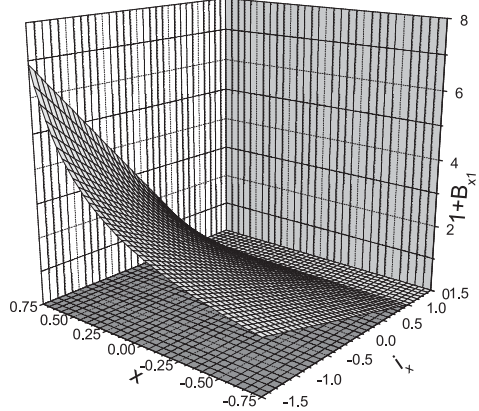

(a)

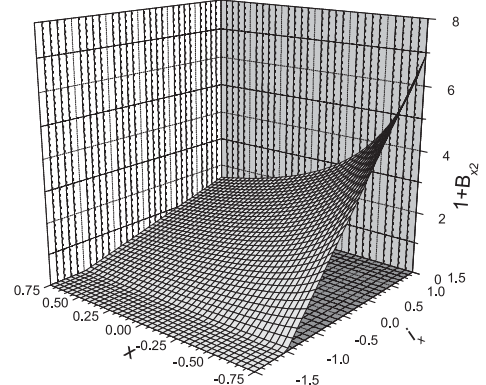

(b)

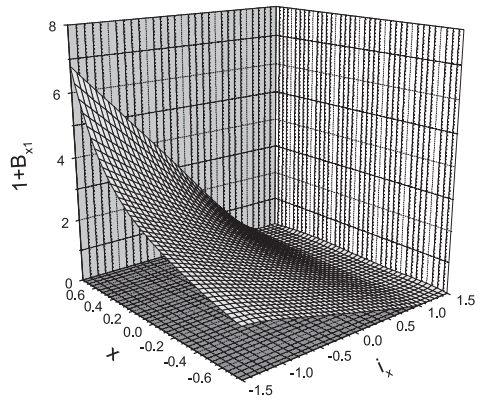

(c)

Fig. 3 Magnetic flux densities in Magnets $x 1$ and $x 2$ considering discontinuous characteristic, and its power series approximation for Magnets $x 1$

Then, the second representation is considered in order to reduce the number of power series terms. To this end, the first term of the right side of the equation Eq.(9) is noted. By introducing the notations $B_{x p}=B_{x 1}+B_{x 2}$ and $B_{x m}=B_{x 1}-B_{x 2}$, Eq.(9) is expressed as:

$$
\left.\begin{array}{l}
\ddot{x}+i_{p} \omega \dot{y}=k_{m 1}\left(2+B_{x p}\right) B_{x m}+e \omega^{2} \cos \omega t \\
\ddot{y}-i_{p} \omega \dot{x}=k_{m 1}\left(2+B_{y p}\right) B_{y m}+e \omega^{2} \sin \omega t
\end{array}\right\}
$$

The governing equations of $B_{x p}$ and $B_{x m}$ are obtained by the calculations of addition and subtraction of the first and second equations in Eq.(10) as:

$$
\begin{aligned}
& B_{x m}+\beta \dot{B}_{x m} \\
& =\left\{\begin{array}{ll}
(1+\delta)\left(\frac{1-i_{x}}{1+\delta-x}\right) \\
(1+\delta)\left(\frac{1-i_{x}}{1+\delta-x}-\frac{1+i_{x}}{1+\delta+x}\right) \\
-(1+\delta)\left(\frac{1+i_{x}}{1+\delta+x}\right)
\end{array},= \begin{cases}(1+\delta)\left(\frac{1-i_{x}}{1+\delta-x}\right) & \left(i_{x}<-1\right) \\
(1+\delta)\left(\frac{1-i_{x}}{1+\delta-x}+\frac{1+i_{x}}{1+\delta+x}\right) & \left(-1<i_{x}<1\right) \\
(1+\delta)\left(\frac{1+i_{x}}{1+\delta+x}\right) & \left(1<i_{x}\right)\end{cases} \right.
\end{aligned}
$$

The governing equations of $B_{y p}$ and $B_{y m}$ in the $y$ direction are also derived in the same manner.

Figures 4(a) and 4(b) show the values of the terms in the right sides of Eq.(14). From the observations of figures, it seems that $B_{x p}$ can be represented by only even order power series functions of $x$ and $i_{x}$, and $B_{x m}$ can be represented by only odd order power series functions of $x$ and $i_{x}$, respectively. In this paper, they are represented up to the 7 th order terms as:

$$
\left.\begin{array}{rl}
B_{x m}+\beta \dot{B}_{x m} & =Q_{10} x+Q_{01} i_{x}+Q_{30} x^{3}+Q_{21} x^{2} i_{x}+Q_{12} x i_{x}^{2}+\cdots+Q_{16} x i_{x}^{6}+Q_{07} i_{x}^{7} \\
2+B_{x p}+\beta \dot{B}_{x p} & =2+Q_{20} x^{2}+Q_{11} x i_{x}+Q_{02} i_{x}^{2}+\cdots+Q_{15} x i_{x}^{5}+Q_{06} i_{x}^{6}
\end{array}\right\}
$$

In this modeling using the introduced notations $B_{x m}, B_{y m}, B_{x p}$ and $B_{y p}$, the number of terms in the AMB is reduced to a half of the case of approximation of Eq.(12) (total number of terms is 


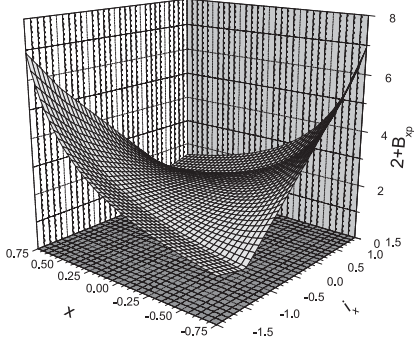

(a)

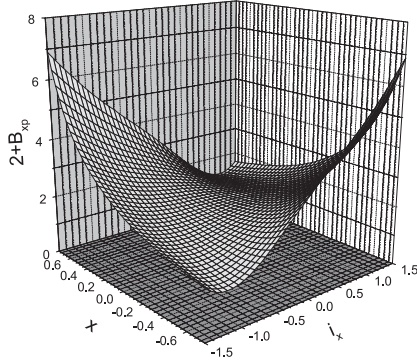

(c)

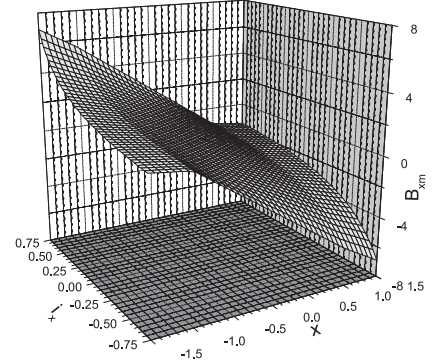

(b)

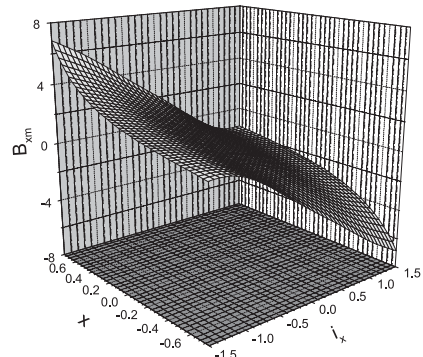

(d)

Fig. 4 Proposed second expression of magnetic flux density in the AMB (considering the discontinuous characteristic), and their power series approximation

reduced from 140 to 70 in the case of the 7 th order approximation). In the theoretical analysis of this paper, Eq.(15) is used as the governing equation of the magnetic flux.

In the coefficients $Q_{i j}$ in Eq.(15), the linear coefficients $Q_{10}$ and $Q_{01}$ are obtained analytically from the Taylor expansion of Eq.(14) for the equilibrium point $\left(x=i_{x}=0\right)$. The other nonlinear coefficients are obtained numerically by using the least square method. These dimensionless values $Q_{i j}$ depend only on the value of $\delta$ because the expressions in the right sides of Eq.(14) have only the parameter $\delta$. When the dimensionless value $\delta$ is set to $\delta=0.141$, which is the average of the values for the experimental setup shown in Table 2, the dimensionless values of the coefficients $Q_{i j}(i, j=1, . ., 7)$ are obtained as:

$$
\begin{aligned}
& Q_{10}=\quad \frac{2}{1+\delta}, Q_{01}=\quad-2, Q_{20}=1.667, Q_{11}=-1.709, Q_{02}=-0.158 \text {, } \\
& Q_{30}=1.430, Q_{21}=-1.120, Q_{12}=-0.151, Q_{03}=-0.267, Q_{40}=0.109 \text {, } \\
& Q_{31}=-1.114, Q_{22}=-0.303, Q_{13}=-0.046, Q_{04}=0.245, Q_{50}=0.052 \text {, } \\
& Q_{41}=-1.394, Q_{32}=-0.257, Q_{23}=-0.382, Q_{14}=0.232, Q_{05}=0.406 \text {, } \\
& Q_{60}=2.310, Q_{51}=-1.837, Q_{42}=0.276, Q_{33}=0.215, Q_{24}=0.192 \text {, } \\
& Q_{15}=0.080, Q_{06}=-0.032, Q_{70}=2.016, Q_{61}=-1.424, Q_{52}=0.211 \text {, } \\
& Q_{43}=0.396, Q_{34}=0.170, Q_{25}=0.152, Q_{16}=-0.036, Q_{07}=-0.099
\end{aligned}
$$

In this calculation of the least square method, the data of the ranges $-1.5<i<1.5$ and $-0.75<x<0.75$ are used. There are some comparatively large coefficients, such as $Q_{60}$ and $Q_{70}$, and they are the coefficients of the terms with high order of the displacement $x$. Because the dimensionless displacement $x$ is usually much smaller than 1, these terms of Eq.(15) become also small. Figures 4(c) and 4(d) show the values of the right sides in Eq.(15). They approximate the values of the right sides in Eq.(14) shown in Figs.4(a) and 4(b) satisfactory.

\section{Theoretical Analysis}

\subsection{Derivation of the Fundamental Equations}

The numerical simulation of the case with the PID control is performed for the system shown in Table 1. Equations (9)-(11) are used for the numerical simulation. Figure 5 shows the resonance curve of the amplitude of shaft displacement at the case of $k_{i}=9.15$. The abscissa 


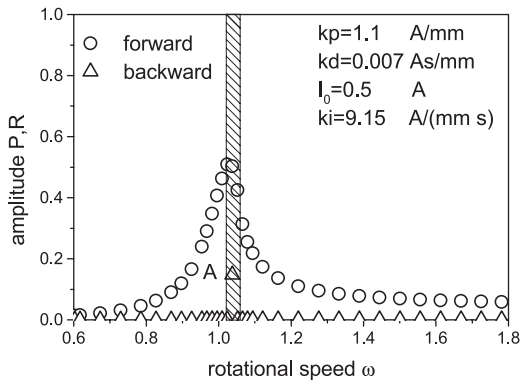

(a)

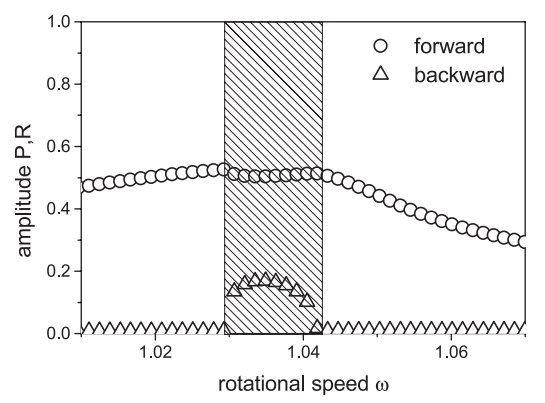

(b)

Fig. 5 Resonance curves of both the forward and backward whirling components

shows the angular velocity $\omega$, which is the dimensionless value to the natural frequency $p$. The ordinate shows the shaft amplitude, which is the dimensionless value to the equilibrium gap $R_{0}$. Both values of $p$ and $R_{0}$ are shown in Table 1 . The symbol $\bigcirc$ denotes the forward harmonic component which whirls in the same direction of the shaft rotation, the symbol $\Delta$ denotes the backward harmonic component. Figure 5(b) shows the occurrence of backward harmonic component at around the major critical speed, and the forward harmonic component reduces its amplitude slightly when the backward harmonic component occurs.

Because such changes of the vibration characteristics can not be clarified by the linear analysis shown in Fig. 2, the nonlinear theoretical analysis is performed. The van der Pol's method $^{(10)(11)}$ is used in the analysis. Figure 6 shows the time histories, orbits, and its spectrum diagrams at around the major critical speed (Point A of Fig.5), and the approximate solution is assumed from its observation. Both forward and backward harmonic components, $\pm \omega$, are considered in the integral of the displacement $s$, the control current $i$, and the magnetic flux density $B_{x m}, B_{y m}$ as their orbits in Fig.6 show the elliptic orbits. Both the constant component and $\pm 2 \omega$ components are considered in the magnetic flux density $B_{x p}$ and $B_{y p}$ from the observation of its spectrum diagram. The solution is assumed as:

$$
\begin{aligned}
& s_{x}=P_{s} \cos \left(\omega t+\delta_{s f}\right)+R_{s} \cos \left(-\omega t+\delta_{s b}\right), \quad s_{y}=P_{s} \sin \left(\omega t+\delta_{s f}\right)+R_{s} \sin \left(-\omega t+\delta_{s b}\right) \\
& i_{x}=P_{i} \cos \left(\omega t+\delta_{i f}\right)+R_{i} \cos \left(-\omega t+\delta_{i b}\right), \quad i_{y}=P_{i} \sin \left(\omega t+\delta_{i f}\right)+R_{i} \sin \left(-\omega t+\delta_{i b}\right) \\
& B_{x m}=P_{B m} \cos \left(\omega t+\delta_{B m f}\right)+R_{B m} \cos \left(-\omega t+\delta_{B m b}\right) \\
& B_{y m}=P_{B m} \sin \left(\omega t+\delta_{B m f}\right)+R_{B m} \sin \left(-\omega t+\delta_{B m b}\right) \\
& B_{x p}=B_{x p 0}+P_{B p} \cos \left(2 \omega t+\delta_{B p f}\right)+R_{B p} \cos \left(-2 \omega t+\delta_{B p b}\right) \\
& B_{y p}=B_{y p 0}+P_{B p} \sin \left(2 \omega t+\delta_{B p f}\right)+R_{B p} \sin \left(-2 \omega t+\delta_{B p b}\right)
\end{aligned}
$$

Here, 18 variables of $P_{s}, \cdots, B_{y a}$ are assumed to be " functions varing slowly with the time". For example, the magnitudes of $\dot{P}_{s}$ and $\ddot{P}_{s}$ are considered as $O(\varepsilon)$ and $O\left(\varepsilon^{2}\right)$, respectively. Here, the symbol $O(\varepsilon)$ expresses the magnitudes of the same order as the small value $\varepsilon$.

The advantages of the proposed second representation of the magnetic flux density, $B_{x m}, \ldots, B_{y p}$, are explained in analytical point of view. When the first representation of $B_{x 1}, \ldots$, $B_{y 2}$ shown in Eq.(10) is used, the constant component, $\omega$ component, and $2 \omega$ component need to be considered in the assumption of each magnetic flux density. Therefore, 5 variables appear for the magnetic flux density of each magnet, $B_{x 1}, \ldots, B_{y 2}$, when they vary independently. For example, the assumed solution of the magnetic flux density for the magnet $x 1$ becomes as

$$
B_{x 1}=B_{x 10}+B_{x 11} \cos \left(\omega t+\delta_{x 11}\right)+B_{x 12} \cos \left(2 \omega t+\delta_{x 12}\right)
$$

Furthermore, there is another advantage in the assumption of solution. The magnetic flux density, $B_{x m}, . ., B_{y p}$, of Eq.(15) shows the behavior almost corresponding the shaft displacement as shown in Fig.6. Therefore, the displacement, the control current, and the magnetic flux density of $B_{x m}$ and $B_{y m}$ can be assumed in the same manner, and $B_{x p}$ and $B_{y p}$ can be represented 

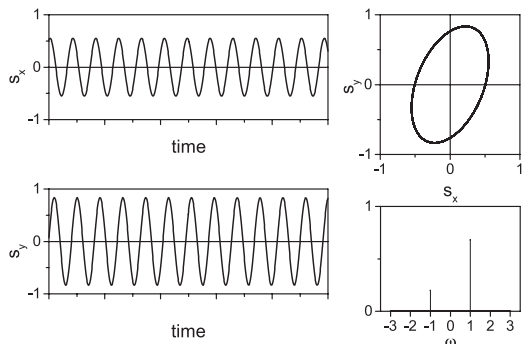

time

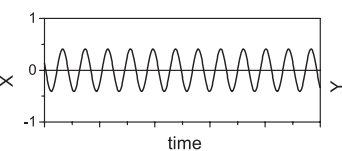

time
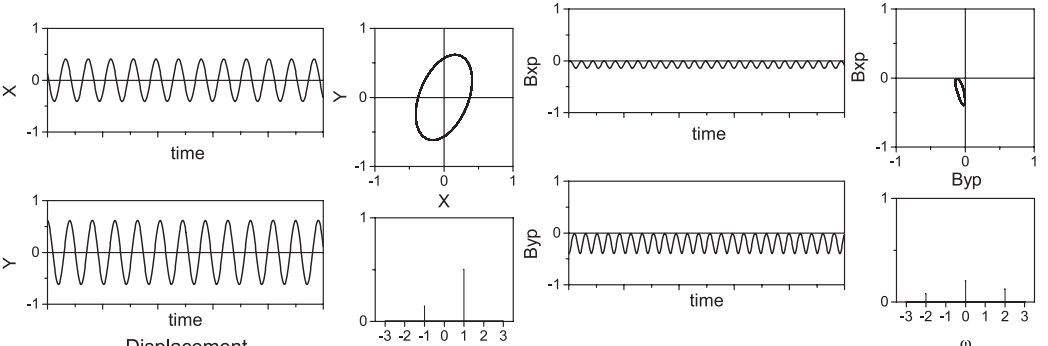

Displacement
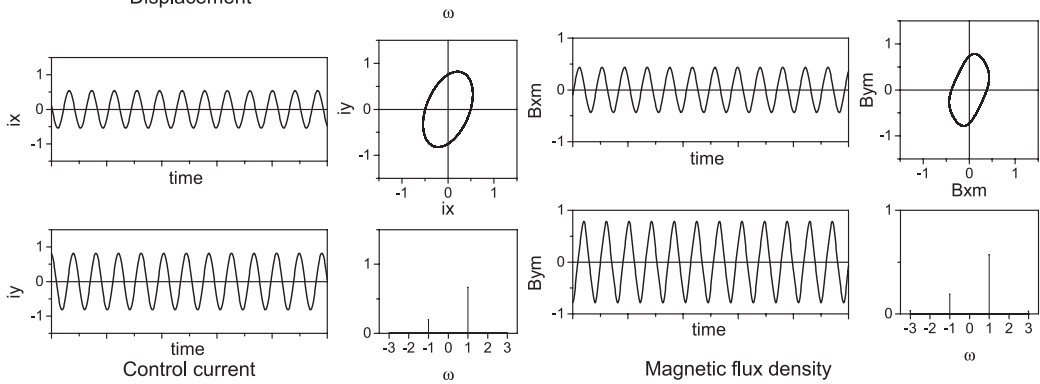

Fig. 6 Time history, Orbit, and spectrum at point A in Fig.5

in the similar manner. According to the considerations above mentioned, the proposed second representation of the magnetic flux density, $B_{x m}, \ldots, B_{y p}$, is appropriate for the dynamical analysis of the rotating shaft supported by the AMB.

Equation (17) is substituted into the equations of motion, Eqs.(11), (13) and (15) for both the $x$ and $y$ directions. The coefficients of $\sin \omega t$ and $\cos \omega t$ on both sides of the six equations, which are Eqs.(11) and (13), and the first equations of Eq.(15) for $B_{x m}$ and for $B_{y m}$, are equated in the accuracy of $O(\varepsilon)$. Furthermore, the constant components and the coefficients of $\sin 2 \omega t$ and $\cos 2 \omega t$ on both sides of the second equations of Eq.(15) for $B_{x p}$ and for $B_{y p}$ are equated in the accuracy of $O(\varepsilon)$. Then, the set of 18 fundamental equations is obtained as follows.

$$
\left.\begin{array}{l}
f_{1}\left(\dot{P}_{s}, \dot{\delta}_{s f}, \cdots, \dot{\delta}_{B p b}, P_{s}, \delta_{s f}, \cdots, \delta_{B p b}\right)=0, \\
\cdots \\
f_{18}\left(\dot{P}_{s}, \dot{\delta}_{s f}, \cdots, \dot{\delta}_{B p b}, P_{s}, \delta_{s f}, \cdots, \delta_{B p b}\right)=0
\end{array}\right\}
$$

The concrete forms of these equations are not shown for the sake of brevity. The steady state solutions, $P_{s 0}, \delta_{s f 0}, \cdots$ and $\delta_{B p b 0}$, are obtained by setting the derivative terms of Eq.(19) equal to 0 as $\dot{P}_{s}=\dot{\delta}_{s f}=\cdots=0$, and by solving these nonlinear algebraic equations. The stability of these obtained steady state solutions is investigated by the eigenvalue analysis.

Figure 7(a) shows the resonance curves of $P=P_{s} \omega$ and $R=R_{s} \omega$ corresponding Fig.5(b), which are the amplitude of both the harmonic component $+\omega$ and backward harmonic component $-\omega$. Lines represent the solution obtained from Eq.(19). The solid line denotes the stable solution, and the dashed line denotes the unstable solution. The circle(O) denotes the harmonic component $P$, and the triangle( $\triangle$ ) denotes the backward harmonic component $R$.

The theoretical analysis explains following: (A) At $\omega<1.030$ or $\omega>1.042$ : The backward component $R$ corresponding the stable harmonic component $P$ is $R=0$. (B) At $1.030<\omega<1.042$ : The stable harmonic component $P$ becomes unstable, and its corresponding backward component $R$ still hold $R=0$. Another stable blanch of the harmonic component $P$ appears due to the bifurcation, and its corresponding backward component $R>0$ appears. 


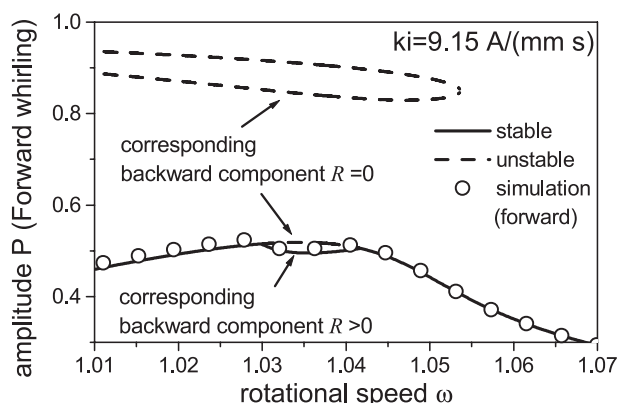

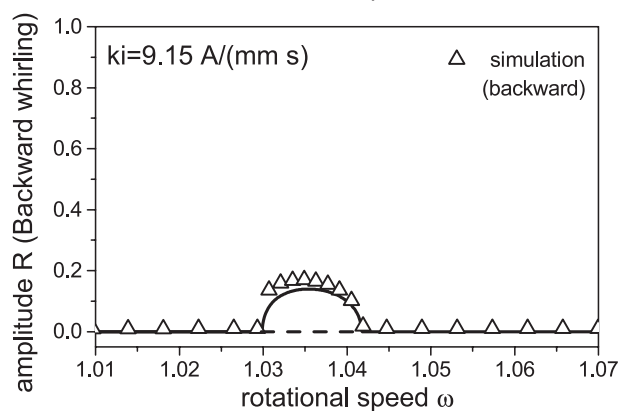

(a) Resonance curve of forward and backward components

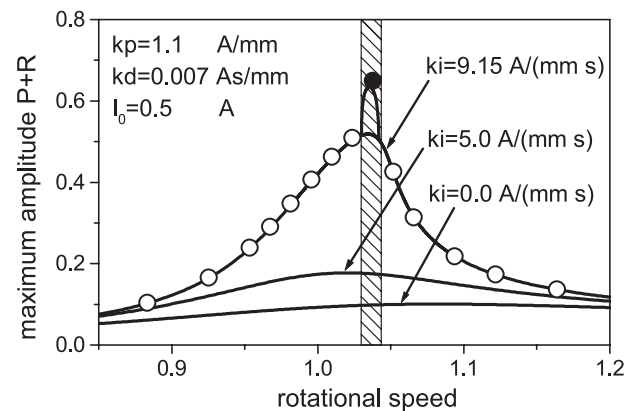

(b) Influence of parameter $k_{i}$

Fig. 7 Resonance curve obtained by the nonlinear theoretical analysis

The theoretical results agree with the numerical results, which confirm the validity of the theoretical analysis.

\subsection{Influence of the integral gain $\boldsymbol{k}_{i}$}

The value of the integral gain $k_{i}$ used in the PID control is usually taken comparatively large in order to eliminate the error of the shaft's equilibrium position caused by the unevenness of the system components. Parameter values shown in Table 1 is used. Figure 7(b) shows the influence of the integral gain $k_{i}$ of the PID control. Because the amplitude of the backward component is $R=0$ at most of the rotational speed ranges, it is difficult to indicate the influence of $k_{i}$ on the backward component by showing both $P$ and $R$ independently. Therefore, this figure represents the maximum amplitude of the orbit, namely $P+R$. The open circle (O) denotes the numerical result of the case with circle orbit, in which only the harmonic component $P$ occurs. The solid circle $(\bullet)$ denotes the numerical result of the case with elliptic orbit, in which both the harmonic and the backward harmonic components occur. As the value of $k_{i}$ increases from 0.0 , the vibration amplitude at the major critical speed increases. The bifurcation of the backward harmonic component $R$ begins to occur when $k_{i}$ is about 5.0 or larger. Figure 7 indicates that the theoretical results including the bifurcation phenomena agree with the numerical results of the piecewise model using Eq.(14). Thus, they clarify the validity of the approximation of power series model shown in Eq.(15), and the procedure of the theoretical analysis.

Furthermore, the authors confirmed in the previous paper that such a bifurcation phenomenon does not occur in the case of PD control for the same system, even if the feedback gains for proportional and derivative, $k_{p}$ and $k_{d}$, are varied ${ }^{(12)}$. Therefore, this bifurcation phenomenon seems to be generated essentially by the influence of the integral feedback.

\section{Experiment}

\subsection{Experimental Setup}

Figure 8(a) shows the experimental setup. A rigid shaft with a circular cross section is simply supported at the upper side by a self-aligning double-row ball bearing and supported at the lower side by a AMB. The material of the shaft is stainless steel, and the shaft's length and the diameter are $l_{s}=600 \mathrm{~mm}$ and $\phi_{s}=20 \mathrm{~mm}$. The positions of disks 1,2 , and 3 from 


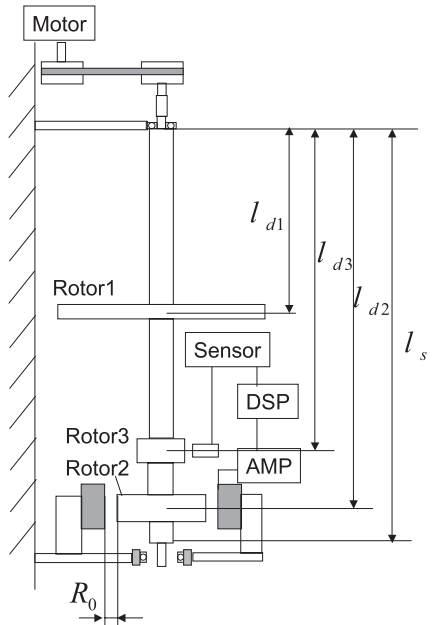

(a)

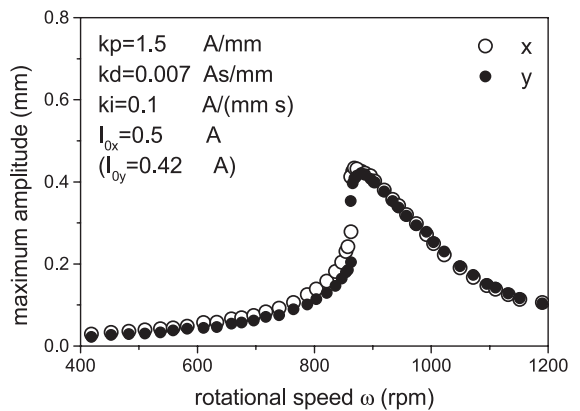

(b)

Fig. 8 Experimental setup, and the resonance curve for the case with slight value of $k_{i}$. (The value of $I_{0 y}$ is tuned to realize $\omega_{x} \simeq \omega_{y}$ )

Table 2 Parameter of electromagnets

\begin{tabular}{|c|c|c|c|c|}
\hline$k_{m}\left[\mathrm{kgmm}^{3} / \mathrm{A}^{2} \mathrm{~s}^{2}\right]$ & $\begin{array}{c}\text { Magnet } x 1 \\
1.19 \mathrm{E}+4\end{array}$ & $\begin{array}{c}\text { Magnet } x 2 \\
1.88 \mathrm{E}+4\end{array}$ & $\begin{array}{c}\text { Magnet } y 1 \\
1.47 \mathrm{E}+4\end{array}$ & $\begin{array}{c}\text { Magnet } y 2 \\
1.69 \mathrm{E}+4\end{array}$ \\
\hline$\delta[\mathrm{mm}]$ & 0.015 & 0.229 & 0.133 & 0.187 \\
\hline
\end{tabular}

the upper end of the shaft are $276 \mathrm{~mm}, 487 \mathrm{~mm}$, and $342.5 \mathrm{~mm}$, respectively. Disk 2 is the rotor disk of the AMB. The shaft displacements in the $x$ and $y$ directions are measured at the position of disk 3 by eddy-current sensors. The diameters and the thicknesses of the disks are $200 \mathrm{~mm}$ and $10 \mathrm{~mm}$ for disk 1, $80 \mathrm{~mm}$ and $30 \mathrm{~mm}$ for disk 2, and $44 \mathrm{~mm}$ and $24 \mathrm{~mm}$ for disk 3, respectively. The radius gap of the AMB is $R_{0}=0.8 \mathrm{~mm}$, and that of the backup bearing is $0.6 \mathrm{~mm}$. The real electromagnetic force shows different characteristic from that of the ideal electromagnet because of the existence of the magnetic saturation, magnetic hysteresis, leakage of the magnetic flux, and so on. This study uses the representation of Eq.(1) for the electromagnetic force, and determines the characteristic constants $k_{m}$ and $\delta$ for each electromagnet experimentally. Table 2 shows the obtained values of constants $k_{m}$ and $\delta$ for all magnets. As these values are different from each other, the critical speeds for the $x$ and $y$ directions, namely $\omega_{x}$ and $\omega_{y}$, have a discrepancy. In order to reduce this discrepancy between $\omega_{x}$ and $\omega_{y}$, the bias current $I_{0 y}$ for the $y$ direction was tuned experimentally with fixing the bias current $I_{0 x}$ for the $x$ direction. Figure $8(\mathrm{~b})$ shows the resonance curve for the case with the tuned bias current $I_{0 y}$. In this case, both the resonance points and the amplitude of the resonance curves for both the $x$ and $y$ direction agree, and the influence of the directional difference is almost vanished.

We note that this resonance curve inclines to the lower speed side more than the case of theoretical analysis. This inclination is affected by the nonlinearity of the magnetic flux density explained in Section 2, and this nonlinearity is influenced by the value of $\delta$ as shown in Eqs.(14) and (15). The actual measured values of $\delta$ have discrepancies, and each measured value may contains the measurement error. These errors affect on the average value used in the theoretical analysis. Additionally, the characteristics such as the magnetic saturation, leakage of the magnetic flux, which are not considered in the modeling may exist in the experimental system, and they affect more on the nonlinear characteristic of the magnetic flux. These points seem to affect on the observed difference of the resonance curve's inclination.

\subsection{Experimental Results}

The fundamental parameters in the experiment are set to $k_{p}=1.1 \mathrm{~A} / \mathrm{mm}, k_{d}=0.007$ As $/ \mathrm{mm}$ and $I_{0 x}=0.5 \mathrm{~A}\left(I_{0 y}=0.42 \mathrm{~A}\right)$. Figure 9 shows the influence of $k_{i}$ on the resonance 


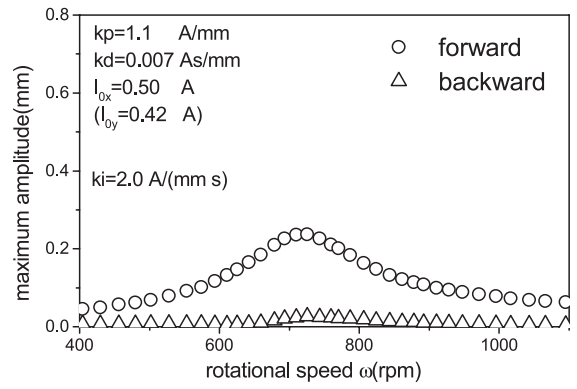

(a)

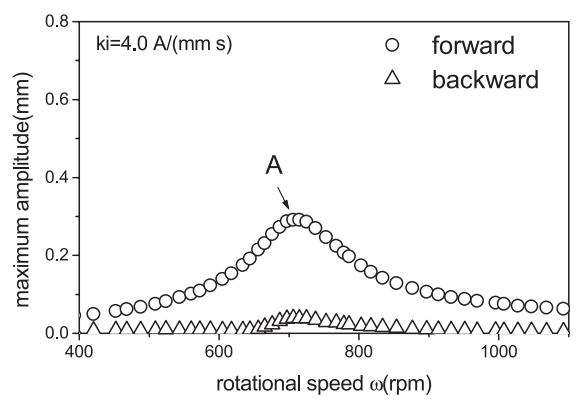

(b)

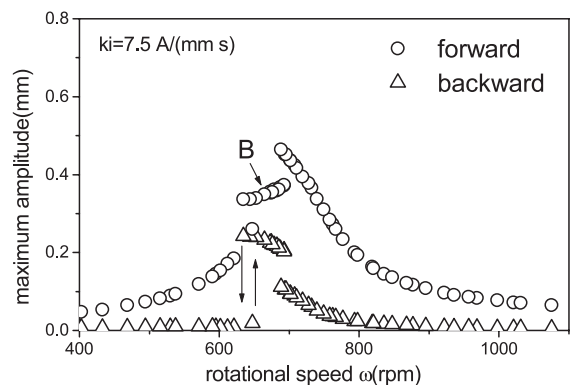

(c)

Fig. 9 Influence of $k_{i}$ (Exp.): $k_{p}=1.1 \mathrm{~A} / \mathrm{mm}, k_{d}=0.007 \mathrm{As} / \mathrm{mm}, I_{0 x}=0.5 \mathrm{~A}$
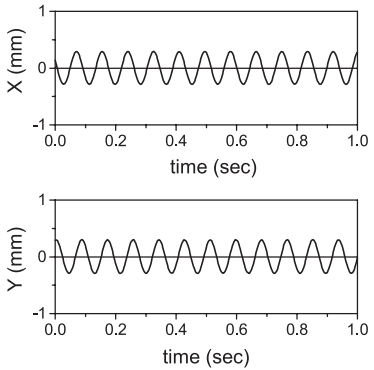

(a) Point $\mathrm{A}$ in the case of $k_{i}=4.0 \mathrm{~A} /(\mathrm{mm} \mathrm{s})$
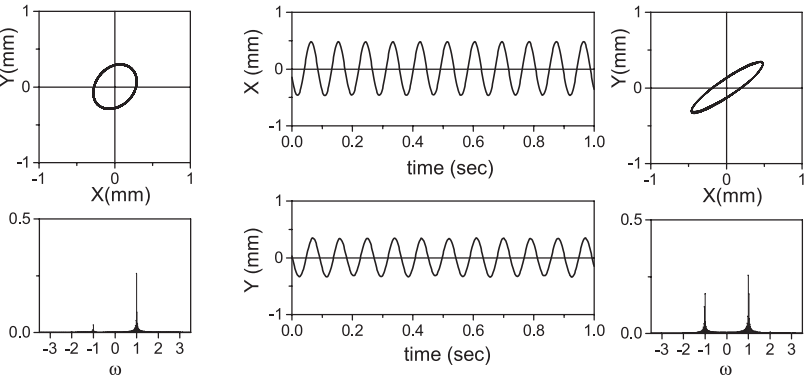

(b) Point $\mathrm{B}$ in the case of $k_{i}=7.5 \mathrm{~A} /(\mathrm{mm} \mathrm{s})$

Fig. 10 Time history, orbit, and spectrum at points A and B in Fig.9 (Exp.)

curve, and Figure 10 shows the time history, orbit and spectrum of the shaft displacement at points A and B in Fig.9. In the resonance curve, the amplitudes of the forward and backward harmonic vibration components are shown with the symbols $\bigcirc$ and $\Delta$, respectively.

The experimental result of Fig.9 corresponds to the theoretical results of Fig.7. As the integral feedback gain $k_{i}$ increases from 2.0 to 4.0 as shown in Figs.9(a) and 9(b), the forward harmonic vibration component $(O)$ increases. Although the backward harmonic vibration component $(\triangle)$ also appear slightly because of the existence of remained directional difference between the $x$ and $y$ directions, the forward harmonic vibration component is dominant as shown in the orbit and spectrum at point A shown in Fig.10(a).

When the value of $k_{i}$ is increased to $k_{i}=7.5$, the forward harmonic vibration component suddenly decreases at around the critical speed. In this rotational speed range, the backward harmonic vibration component $(\triangle)$ becomes large simultaneously. As the result, the orbit changes to elliptic orbit as shown in Fig.10(b) in this range. Because of the softening effect in the resonance curve, the jump phenomenon and the hysteresis phenomenon were observed in the lower speed side of the resonance peak.

As the result, the influence of both the integral feedback and the system nonlinearity on the vibration of the rotor system supported by the AMB is confirmed experimentally.

\section{Conclusion}

The vibration of the rigid rotating shaft supported by the PID controlled AMB is consid- 
ered. The current control type power amplifier is used, and its PI control characteristic and the delay of the magnetic flux in the electromagnet are noted. The modeling of the system is performed, and the influences of the dynamics of both electric and magnetic circuits are investigated by the nonlinear theoretical analysis. Following results are obtained:

(1) The nonlinear power series approximation model for the magnetic flux of the AMB is proposed utilizing the symmetry of the magnets arrangement in the AMB. It enables us to reduce both the number of the approximation terms and the number of the variables in the analysis. Therefore it makes the nonlinear theoretical analysis much simpler.

(2) The procedure of the nonlinear vibration analysis of the rotor system supported by the AMB with the PID controller is developed, and the nonlinear dynamical characteristics of the rigid rotor system supported by the AMB with the PID controller are clarified theoretically. Although the closed and concise form of the approximated solution is not represented and the approximated solution still needs the numerical calculation, it enables us to perform the various investigation, such as the bifurcation analysis and the optimization of the parameters considering nonlinear effect, easier than using the direct numerical simulation.

( 3 ) The influence of the integral feedback gain is investigated theoretically and experimentally. When the gain of the integral feedback control increases, the backward harmonic component is caused in the vicinity of the critical speed due to the nonlinear bifurcation.

\section{References}

( 1 ) G.Schweitzer, H.Bleuler, A.Traxler, Active magnetic bearings, Hochschulverlag AG an der ETH, Zürich

( 2 ) The JSME ed., The basis and application of the magnetic bearings, Yokendo co.,ltd.,(1995) (in Japanese)

( 3 ) S.C.Chang and P.C.Tung, Nonlinear Identification of a Magnetic Bearing System with Closed Loop Control”, JSME International Journal, Series C, Vol.42, No.4, 1999, pp.982-990.

( 4 ) M. Chinta and A. B. Palazzolo, Stability and Bifurcation of Rotor Motion in a Magnetic Bearing, Journal of Sound and Vibration, Vol.214,No.5 (1998), pp.793-803

( 5 ) J.C.Ji L.Yu, and A.Y.T.Leung, Bifurcation Behavior of a Rotor Supported by Active Magnetic Bearing, Journal. of Sound and Vibration, Vol.235,No.1 (2000), pp.133-151

( 6 ) J.C.Ji and C. H. Hansen, Non-linear Oscillations of a Rotor in Active Magnetic Bearing, Journal of Sound and Vibration, Vol.240,No.4 (2001), pp.599-612

( 7 ) J.C.Ji and A.Y.T.Leung, Non-linear Oscillation of a Rotor-Magnetic Bearing System Under Superharmonic Resonance Conditions, International Journal of Non-linear Mechanics, Vol.38,No.6 (2003), pp.829-835

( 8 ) J.C.Ji, Stability and Hopf Bifurcation of a Magnetic Bearing System With Time Delays, Journal of Sound and Vibration, Vol.259,No.4 (2003), pp.845-856

( 9 ) Y.S.Ho, H.Liu, and L.Yu, Effect of Thrust Magnetic Bearing on Stability and Bifurcation of a Flexible Rotor Active Magnetic Bearing System, Transaction of ASME, Journal of Vibration and Acoustics, Vol.125,No.3 (2003), pp.307-316

(10) T.Yamamoto and Y.Ishida, Linear and Nonlinear Rotordynamics, John Wiley $\mathcal{E}$ Sons, Inc.,(2001)

(11) Stoker, J.J., Nonlinear Vibrations in Mechanical and Electrical Systems, John Wiley $\mathcal{E}$ Sons, Inc., (1950)

(12) T.Inoue, Y.Sugawara, M.Sugiyama, Modelling and Nonlinear Vibration Analysis of a Rigid Rotor System supported by the Magnetic Bearing (Effects of Delays of Both Electric Current and Magnetic Flux), Transaction of ASME, Journal of Applied Mechanics, Vol.77,(2010) 011005. 